\title{
HALF-CUTTING METHOD during Hysterectomy for Large Uterine Cervical Myoma
}

\author{
Yosuke Fukui', Yuki Yamada', Seiji Mabuchi² \\ ${ }^{1}$ Department of Obstetrics and Gynecology, Nara Medical University, Kashihara, Japan \\ ${ }^{2}$ Department of Gynecology, Osaka International Cancer Institute, Osaka, Japan \\ Email: seiji.mabuchi@oici.jp
}

How to cite this paper: Fukui, Y., Yamada, Y. and Mabuchi, S. (2021) HALF-CUTTING METHOD during Hysterectomy for Large Uterine Cervical Myoma. Open Journal of Obstetrics and Gynecology, 11, 1196-1201. https://doi.org/10.4236/ojog.2021.119113

Received: August 21, 2021

Accepted: September 14, 2021

Published: September 17, 202

Copyright $\odot 2021$ by author(s) and Scientific Research Publishing Inc. This work is licensed under the Creative Commons Attribution International License (CC BY 4.0).

http://creativecommons.org/licenses/by/4.0/

\begin{abstract}
Hysterectomy for large uterine cervical myoma is a challenging surgical procedure due to the limited operative field for lateral and posterior dissections. Existing procedures such as performing myomectomy before hysterectomy or performing retrograde hysterectomy remain suboptimal in expanding the operative field, especially in cases with a huge cervical myoma. In this report, we introduce a new procedure, the "HALF-CUTTING METHOD" which can be used to obtain an adequate surgical field during hysterectomy.
\end{abstract}

\section{Keywords}

HALF-CUTTING METHOD, Cervical Myoma, Hysterectomy, Surgical Field

\section{Introduction}

Uterine leiomyomas are the most common pelvic tumors in women, with a prevalence of $20 \%-40 \%$ after the age of 35 years [1]. Of these cases, $95 \%$ occur in the uterine corpus while a frequency of only $0.6 \%$ is reported for cervical leiomyomas [2].

Cervical myomas differ from their corpus counterparts, where symptoms of abnormal uterine bleeding predominate. Due to the lack of menstrual symptoms, cervical myomas usually grow to large sizes before detection. The size of cervical myoma in previous reports ranges from $5-24 \mathrm{~cm}$ [3].

Surgery remains the mainstay of treatment for most leiomyomas. Indications for surgical intervention include tumor-related bulk symptoms, abnormal uterine bleeding, recurrent pregnancy loss, and infertility. In postmenopausal women or premenopausal women who are not desirous of preserving their fertility, hysterectomy is the standard treatment as it eliminates both the symptoms and the possibility of future recurrence. 
Hysterectomy is challenging to perform in cases with a large cervical myoma due to the limited operative field for the complete incision of the parametrium. For such cases, previous reports have suggested performing myomectomy before hysterectomy or performing retrograde hysterectomy to expand the operative field; however, these procedures are far from optimal. Thus, it is important to develop new methods to supplant these procedures when they are inefficient or unsuccessful.

In this report, we introduce a new procedure called the "HALF-CUTTING METHOD" which can be used to obtain an optimal surgical field for lateral and posterior dissections during hysterectomy for large cervical myoma.

\section{Surgical Procedures}

Total abdominal hysterectomy using the HALF-CUTTING METHOD can be safely performed according to the method described below.

1) Perform the laparotomy, identify the major uterine ligaments, and check the mobility of the uterus.

2) Incise the round ligaments and the peritoneum of the vesicouterine pouch, and dissect the bilateral pelvic sidewall triangles, which are surrounded by the round ligament, infundibulopelvic ligament, and posterior lobe of the broad ligament.

3) Incise the ovarian ligaments and fallopian tubes (or infundibulopelvic ligaments).

4) After the development of paravesical spaces, identify the uterine artery, which can be traced from the internal iliac artery, and incise it at its origin.

5) Push the bladder down from the anterior wall of the cervix and vagina as much as possible, and identify the anterior vaginal fornix by manual examination in the vaginal canal, then incise the anterior vaginal wall. In cases where the posterior vaginal fornix was visible, circumferential dissection of the vagina is done while carefully avoiding rectal injury. By performing stapes $3-6$, the uterine blood flow can be significantly reduced.

6) The uterus is then cut in half from the fundus to the cervix (cervical myoma). Crossing the half-cut uterus reduces the width of the uterus (Figure 1 and Figure 2) and allows the surgeon to obtain the optimal surgical field for lateral and posterior dissections.

7) Identify and isolate the ureters from the posterior leaf of the broad ligament to the ureteral tunnel. Incise the posterior leaf of the broad ligaments and uterosacral ligaments. By changing the position of the half-cut uterus, the parametrium can be fully exposed, coagulated and incised.

8) In cases wherein circumferential dissection of the vagina could not be performed in step 6 , colpotomy is completed by cutting the posterior vaginal wall. Then excise the remaining connective tissues surrounding the vagina and remove the uterus.

9) Suture the vaginal vault. 

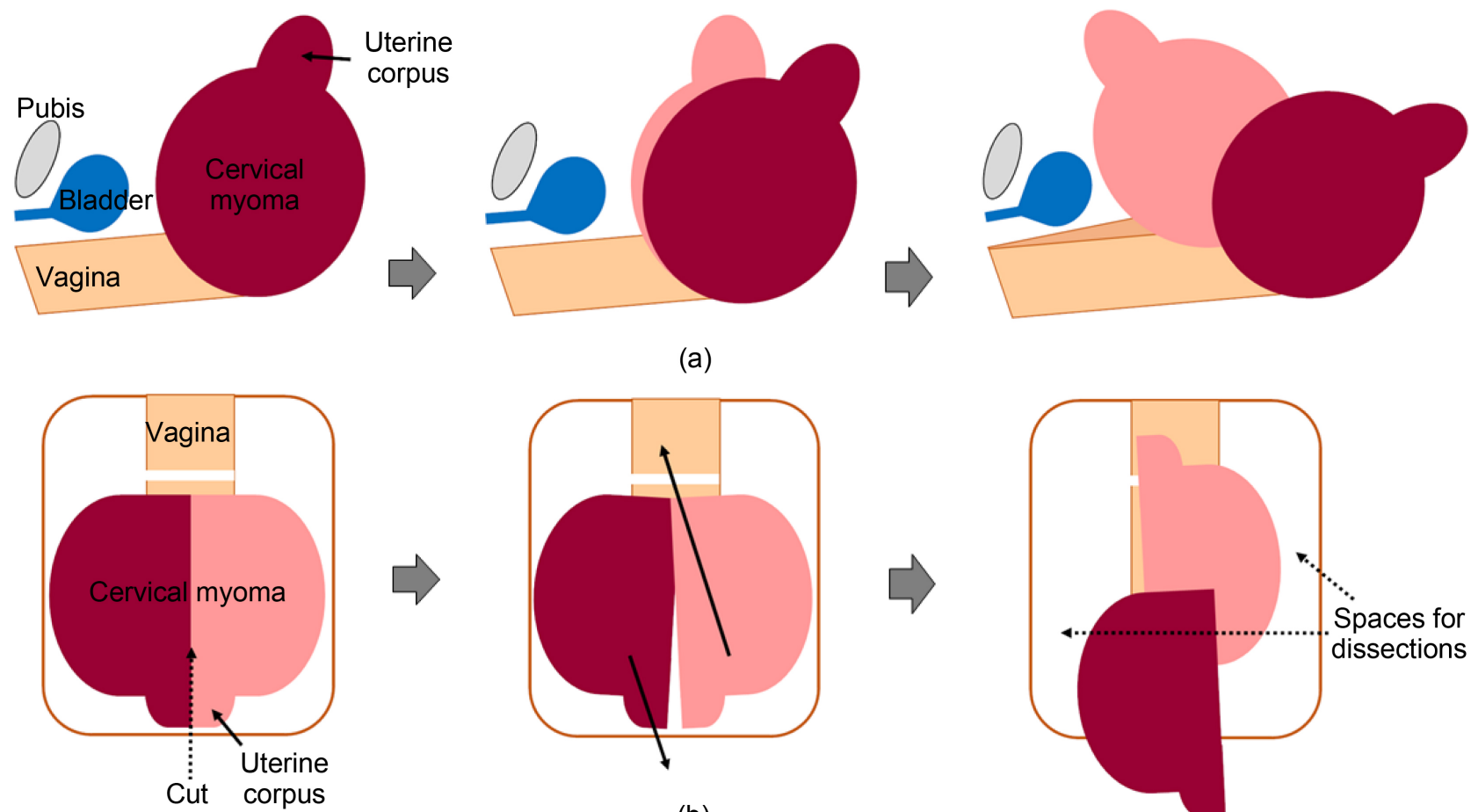

(a)
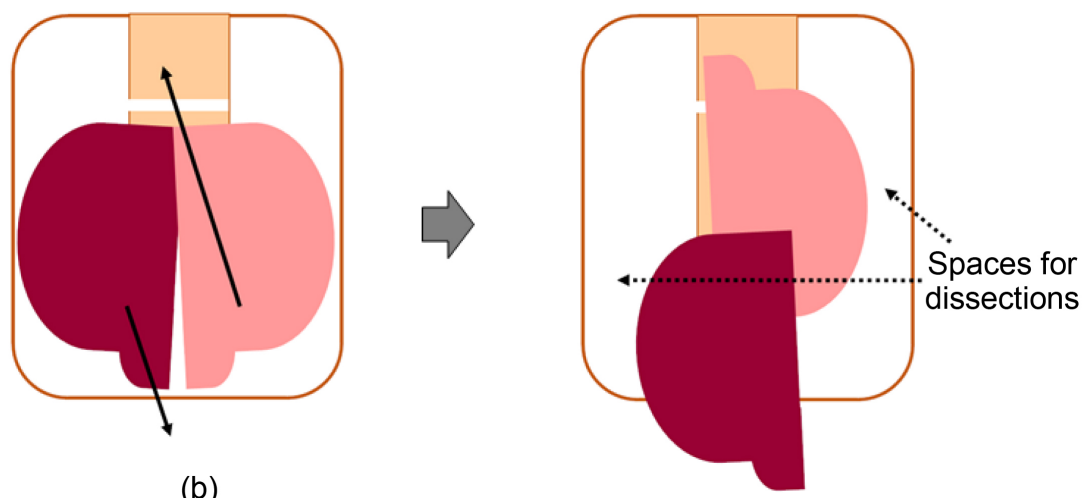

(b)

Figure 1. Schematic diagram of the HALF-CUTTING METHOD. (a) Longitudinal view, (b) Frontal view.

(i)

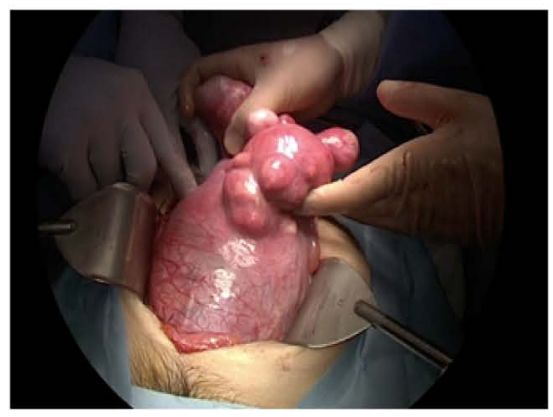

(iii)

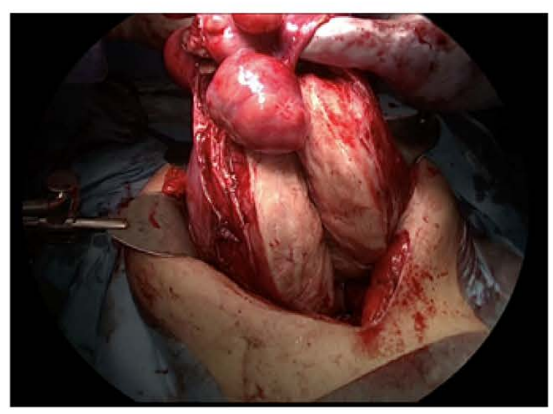

(ii)

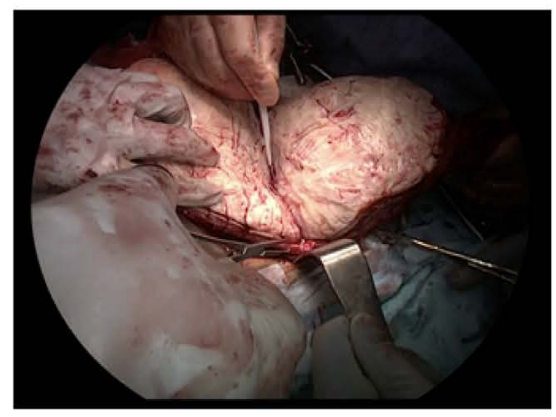

(iv)

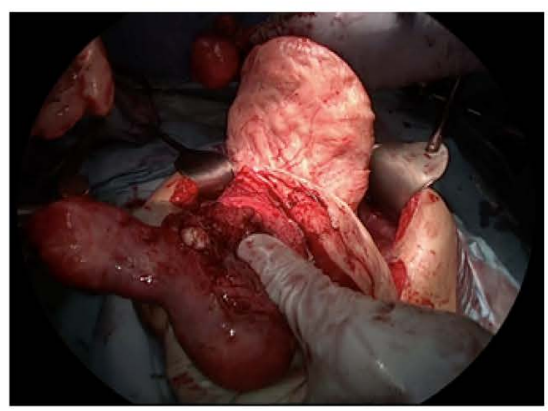

(v)

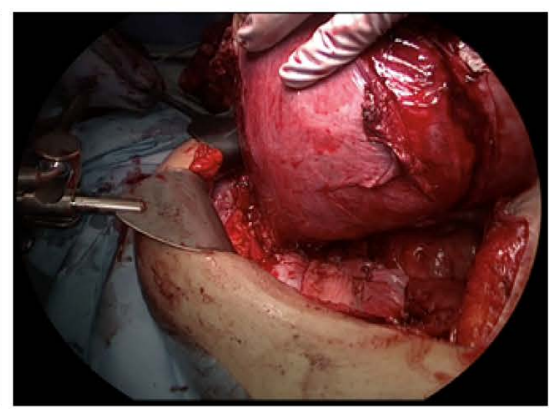

Figure 2. Hysterectomy using the HALF-CUTTING METHOD, shown in representative photos. (i), large cervical myoma occupying surgical field. (ii)-(iii), the uterus was cut in half from the fundus to the cervix. (iv)-(v), the uterine width was reduced by crossing the half-cut uterus, and optimal surgical field for lateral and posterior dissections was obtained. 


\section{Case Presentation}

A 49-year-old nulliparous (G0P0) Japanese woman presented with abdominal distension. Cytology of the cervix was negative for intraepithelial lesions of malignancy (NILM). MRI revealed an 18 by 15 by $11 \mathrm{~cm}$ leiomyoma in the cervix and $4 \mathrm{~cm}$ and $5 \mathrm{~cm}$ leiomyomas in the uterine corpus. Her past surgical and medical histories were unremarkable. After discussing treatment options, a total abdominal hysterectomy and bilateral salpingectomy were performed. During hysterectomy, an enlarged uterine cervix obstructed the surgical field for lateral and posterior dissections; thus, the HALF-CUTTING METHOD was employed (Figure 2). The procedure lasted for 219 minutes, with intraoperative blood loss of $500 \mathrm{~mL}$. The weight of the removed uterus was $2454 \mathrm{~g}$ and pathological analysis confirmed leiomyoma. The postoperative course was uneventful, and the patient was discharged on postoperative day 8 without complications.

\section{Discussion}

In this report, we introduce a new procedure, termed "HALF-CUTTING METHOD" which can be used during hysterectomy for large cervical myoma.

Large cervical myomas pose challenges during hysterectomy, as the size adds obstruction in the surgical field and complicates lateral or posterior dissections. To our knowledge, two different options have been proposed to safely complete hysterectomy for cervical myoma, namely, performing myomectomy before hysterectomy or performing retrograde hysterectomy [4] [5]. Although these procedures can help expand the operative field in some cases, myomectomy before lateral dissection can cause massive bleeding in cases involving an extremely large cervical myoma. Moreover, performing retrograde hysterectomy often does not sufficiently expand the field for the visualization of ureters, thereby impeding the safe incision of the parametrium, uterosacral ligaments, and the posterior leaf of broad ligaments.

We believe that the use of the HALF-CUTTING METHOD has important clinical implications. Firstly, this method does not require additional surgical devices. Secondly, it can be performed even in cases wherein the ureters are unidentifiable behind the huge cervical myoma. This method requires only the identification of the uterine artery origin from the internal iliac artery and of the anterior vaginal fornix, which are not difficult to achieve. The uterine artery can be identified by tracing the internal iliac artery from paravesical spaces, which easily develop even in cases with large cervical myoma. Additionally, the anterior vaginal fornix can easily be identified at the lower end of the cervical myoma, which causes the stretching and upward lift of the vaginal fornixes; hence, cutting the anterior vaginal wall can be safely performed. Thirdly, performing this procedure for cervical myoma significantly reduces the width of the uterus by up to $50 \%$, which provides enough space for the identification of ureters and subsequent safe dissection of the posterior and lateral uterine components. 
Although no complications were observed in our cases, we recognize some potential risks related to the use of the HALF-CUTTING METHOD. Hemorrhage may occur from a vaginal wall that was incised before the completion of lateral and posterior dissections. However, such vaginal wall bleeding is usually mild, as demonstrated in previous studies on retrograde hysterectomy [6]. There is another risk for hemorrhage from the half-cut uterine corpus and cervical myoma. This may be minimized by blocking the blood flow through ligation of the uterine and ovarian arteries. The blood flow from the vagina can also be significantly blocked by cutting the anterior vaginal wall or both the anterior and posterior vaginal walls; thus, serious hemorrhage from the half-cut uterine corpus and cervical myoma rarely occur.

In conclusion, we developed a new procedure, the "HALF-CUTTING METHOD" which can be employed during hysterectomy for large cervical myoma. We believe that this procedure enables surgeons to obtain an optimal surgical field and eliminate the possibility of ureteral injury or massive hemorrhage. This preliminary but promising result justifies further investigation of this procedure in larger prospective studies.

\section{Ethical Approval Status}

The Ethics Committee of Nara Medical University decided that this case report does not require IRB approval due to the nature of this study: a report describing the treatment of a single patient and thus does not meet the definition of human subjects research.

\section{Conflicts of Interest}

The authors declare no conflicts of interest.

\section{References}

[1] Laganà, A.S., Alonso Pacheco, L., Tinelli, A., Haimovich, S., Carugno, J., Ghezzi, F., Mazzon, I. and Bettocchi, S. (2019) Management of Asymptomatic Submucous Myomas in Women of Reproductive Age: A Consensus Statement from the Global Congress on Hysteroscopy Scientific Committee. Journal of Minimally Invasive Gynecology, 26, 381-383. https://doi.org/10.1016/j.jmig.2018.06.020

[2] Tiltman, A.J. (1998) Leiomyomas of the Uterine Cervix: A Study of Frequency. International Journal of Gynecological Pathology, 17, 231-234. https://doi.org/10.1097/00004347-199807000-00006

[3] Wong, J., Tan, G.H.C., Nadarajah, R. and Teo, M. (2017) Novel Management of a Giant Cervical Myoma in a Premenopausal Patient. BMJ Case Reports, 2017, bcr2017221408. https://doi.org/10.1136/bcr-2017-221408 https://casereports.bmj.com/content/casereports/2017/bcr-2017-221408.full.pdf

[4] Hiramatsu, Y. (2019) Hysterectomy for Cervical and Intraligamental Fibroids. Surgery Journal, 6, 2-10. https://doi.org/10.1055/s-0039-1698419

[5] Ishidera, Y., Furugori, M., Hirata, G., Wakabayashi, R., Shigeta, H. and Yoshida, H. (2021) Total Laparoscopic Hysterectomy for Anterior Cervical Myoma: Possible Significance of Presurgical Assessment by Magnetic Resonance Imaging. Gynecol- 
ogy and Minimally Invasive Therapy, 10, 61-64.

[6] Hiramatsu, Y. (2019) Retrograde Abdominal Hysterectomy. Surgery Journal, 5, 27-32. https://doi.org/10.1055/s-0039-1683919 